\section{A Função Gonadal do Homem Obeso}

\begin{abstract}
RESUMO
Em homens obesos, os niveis séricos de globulina ligadora de hormônios sexuais (GLHS), bem como de testosterona total, (TT) estão diminuídos. Dados relativos aos niveis séricos de testosterona livre (TL) são controversos. Homens com obesidade mórbida apresentam valores plasmáticos de testosterona livre diminuídos, levando à alteração dos mecanismos de retro-regulação. O decréscimo funcional da amplitude do pulso de $\mathrm{LH}$, bem como a diminuição dos niveis de $L H$, seriam a causa do hipoandrogenismo. Estudamos dois grupos de homens obesos: Grupo $1\left(\mathrm{IMC} \leq 35 \mathrm{~kg} / \mathrm{m}^{2}\right)$ e Grupo $2\left(\mathrm{IMC} \geq 35 \mathrm{~kg} / \mathrm{m}^{2}\right)$ antes e após seis meses de dieta hipocalórica (1.200Kcal/dia). Todos os pacientes utilizaram dexfenfluramina $(15 \mathrm{mg} 2 x$ ao dia) durante todo o período do estudo. Niveis plasmáticos de insulina, bem como valores séricos de testosterona total, testosterona livre e LH foram dosados antes e após a perda de peso. Pacientes com obesidade moderada $\left(I M C=32,3 \pm 1,9 \mathrm{~kg} / \mathrm{m}^{2}\right)$ apresentaram diminuição significativa na concentração sérica de TT (390 $\pm 120 \mathrm{ng} / \mathrm{dL})$, bem como de $T L$ (média $\pm \mathrm{DP} 16,0 \pm 4,8 \mathrm{pg} / \mathrm{mL}$ ) quando comparados a normais. A concentração de LH sérico $(4,5 \pm 2,9 \mathrm{mlU} / \mathrm{mL})$ foi considerada normal. Niveis de insulina mostravam-se elevados em todos os pacientes (46,3 $\pm 30,1 \mu \mathrm{U} / \mathrm{mL}$ ). Após a perda de peso, notou-se aumento significante $(p<0,01)$ dos níveis séricos de $\mathrm{LH}, \mathrm{TT}$ e TL concomitante à queda da concentração de insulina plasmática. Em homens com obesidade de grande porte $\left(\mathrm{IMC}=43,0 \pm 6,7 \mathrm{~kg} / \mathrm{m}^{2}\right)$, os niveis de $\Pi(320 \pm 110 \mathrm{ng} / \mathrm{dL})$, $\mathrm{TL}(11,0 \pm 2,1 \mathrm{pg} / \mathrm{mL})$ e $\mathrm{LH}(3,1 \pm 1,3 \mathrm{mlU} / \mathrm{mL})$ mostraram-se significativamente menores quando comparados ao Grupo 1 e aos controles normais. Como esperado, após a perda de peso, os níveis séricos de TT, $\mathrm{TL}$ e LH aumentaram significativamente enquanto a concentração plasmática de insulina diminuiu. Concluímos que os níveis de TL são dependentes do grau de obesidade: homens com obesidade de grande porte (IMC $\left.\geq 35 \mathrm{~kg} / \mathrm{m}^{2}\right)$ são considerados como candidatos a apresentarem baixos valores séricos de TL. (Arq Bras Endocrinol Metab 2000; 44/1: 31-7)
\end{abstract}

Unitermos: Testosterona; Hipogonadismo: Obesidade; Perda de peso; Insulina: Gonadostato

\begin{abstract}
In obese men, sex hormone-binding globulin (SHBG) as well as total testosterone (TT) levels are decreased. Data concerning serum free testosterone (FT) levels in obese men are discordant. FT levels are decreased in only some morbidly obese men consistent with an impairment of the feedback regulatory mechanism. It has been suggested that a functional decrease of LH pulse amplitude and serum LH levels are reflected in their hypoandrogenism. We have studied two groups of obese men (Group 1: BMI $\leq 35 \mathrm{~kg} / \mathrm{m}^{2}$ and Group 2: BMI $\geq 35.1 \mathrm{~kg} / \mathrm{m}^{2}$ ) before and after six months of a low calorie diet (1200kcal/day). Every patient received a therapeutic prescription of
\end{abstract}

\section{artigo original}

\author{
Nicolau Lima \\ Humberto Cavaliere \\ Alfredo Halpern \\ Geraldo Medeiros-Neto
}

Unidade de Tireóide e Grupo Geral, Disciplina de Endocrinologia, Faculdade de Medicina da Universidade de São Paulo, SP. 
dexfenfluramine (15mg b.i.d.) that was maintained for six months. Plasma insulin levels, serum total testosterone, free testosterone and LH concentrations were obtained before and after weight loss. Moderately obese men (BMI $=32.3 \pm$ $1.9 \mathrm{~kg} / \mathrm{m}^{2}$ ) presented significantly decreased TT levels $(390 \pm 120 \mathrm{ng} / \mathrm{dL}$ ) as well as FT (mean \pm SD: $16.0 \pm 4.8 \mathrm{pg} / \mathrm{mL}$ ) as compared with normal controls. Serum LH concentrations $(4.5 \pm 2.9 \mathrm{~m} / \mathrm{U} / \mathrm{mL})$ were normal. Insulin levels were elevated in all patients $(46.3 \pm 30.1 \mu \mathrm{U} / \mathrm{mL})$. After weight-loss there was a significant $(p<0.01)$ increase in TT, FT and LH levels whereas insulin concentrations significantly decreased. In massively obese men (BMI $=43.0 \pm$ $\left.6.7 \mathrm{~kg} / \mathrm{m}^{2}\right)$ TT $(320 \pm 110 \mathrm{ng} / \mathrm{dL})$. FT $(11.0 \pm 2.1 \mathrm{pg} / \mathrm{mL})$ and $\mathrm{LH}(3.1 \pm 1.3 \mathrm{mlU} / \mathrm{mL})$ were decreased and significantly lower as compared with the previous group and normal controls. As expected, after weight loss as expected, TT. FT and LH levels increased significantly while insulin concentrations decreased. We concluded that FT levels are dependent on the degree of obesity, massively obese men (BMI $\geq 35.1 \mathrm{~kg} / \mathrm{m}^{2}$ ) being considered as candidates for consistently low FT levels. (Arq Bras Endocrinol Metab 2000; 44/1: 31-7)

Keywords: Testosterone: Hypogonadism: Obesity: Weight loss; Insulin; Gonadostate

V Ários relatos (1-10) Na iLTERATURa vêm indicando que níveis séricos de testosterona total em homens obesos mostram-se, quase sempre, em valores abaixo da normalidade quando comparados a homens normais na mesma faixa etária.

Além disso, também foi observado por diversos pesquisadores, decréscimo na capacidade de ligação da globulina carregadora de hormônios sexuais (SHBG) em homens obesos comparativamente à população masculina de peso normal e dentro da mesma faixa et.ria $(1-4,6-8)$.

Muitos estudos (14-20) têm demonstrado uma relação inversa entre as concentrações séricas de insulina e globulina ligadora de hormônios sexuais (SHBG) nas mulheres obesas, porém, pouco se investigou esta relação em homens obesos. Tem sido sugerido que esta relação pode estabelecer-se por um efeito inibitório da insulina na síntese de SHBG. Este efeito foi demonstrado in vitro em células de hepatoma humano (21), mas não in vipo. Por outro lado, como a concentração de SHBG varia inversamente $(22-25)$ e a concentração de insulina varia diretamente $(26,27)$ com o grau de obesidade, tem sido sugerido que a aparente relação inversa entre
SHBG e níveis de insulina pode simplesmente refletir a relação de ambas as variáveis com o grau de obesidade.

Vermeulen et al. (1996), estudando grupo de homens obesos, verificaram que os níveis de SHBG correlacionaram-se inversamente com os níveis de insulina, IGF-1 e concentração de GH em 24 horas. Após obter redução no peso (cerca de $15 \mathrm{~kg}$ ), verificaram que havia aumento na capacidade ligadora do SHBG para valores normais, mesmo com os pacientes ainda apresentando peso elevado e níveis séricos de insulina aumentados.

A queda do nível de testosterona total estaria ligada a menor disponibilidade de SHBG, levando-se a pensar que a possível diminuição de testosterona livre sérica seria de menor porte ou inexistente. Realmente, enquanto três grupos de pesquisadores (1-3) observaram níveis séricos normais de testosterona livre na obesidade masculina, outros quatro trabalhos $(4,5,7)$ indicaram que a testosterona livre apresentava baixas concentrações séricas em obesos de grande porte (obesidade mórbida).

Os mecanismos responsáveis pelo decréscimo da testosterona livre nesses pacientes ainda são parcialmente desconhecidos. Especificamente, ainda deveríamos definir se este relativo hipoandrogenismo seria ligado à falha primária de função gonadal (testicular) ou seria devido ao mecanismo central, hipotalâmico-hipofisário. No sentido de afastar a hipótese gonadal, demonstrou-se que o estímulo de células de Leydig por doses farmacológicas de hCG (gonadotrofina coriônica) é normal nos obesos de grande porte $(1,28)$, sugerindo-se que a causa primária da diminuição de testosterona livre não estaria vinculada à produção gonadal do hormônio masculino. Por outro lado, vários estudos indicam que os níveis séricos de gonadotrofinas são normais no homem obeso, embora possam decrescer nas obesidades de grande porte, sugerindo, possivelmente, uma origem central para o decréscimo de testosterona livre.

Para melhor avaliar o papel de LH-FSH no hipoandrogenismo do homem obeso, vários grupos de pesquisadores avaliaram os níveis séricos de gonadotrofinas em homens obesos $(1,5,18,29-30)$. Um desses trabalhos demonstrou níveis subnormais de FSH sérico (30) e os demais mostraram níveis séricos de FSH normais.

O LH sérico foi avaliado de forma mais ampla por Vermeulen et al. (1993) e Giagulli et al. (1994), onde foi estudada a pulsatilidade das gonadotrofinas em homens obesos. Confirma-se o nível médio baixo 
de LH sérico, queda da média dos pulsos de amplitude de LH, bem como da soma das amplitudes. Tais fenômenos confirmam menor massa secretória gonadotrófica, conduzindo a confirmação de disfunção hipotálamo-hipofisária.

Notou-se, ainda, correlação significativa entre a soma da amplitude dos pulsos gonadotróficos e os valores de testosterona livre. A queda do hormônio gonadal (testosterona) livre seria devido a comprovada atenuação dos pulsos gonadotróficos hipofisários.

Tal fenômeno poderia ser condicionado a:

1. Niveis relativamente elevados de estradiol no homem obeso. Todavia, em outros trabalhos $(4,10)$ não foi observada correlação positiva e significativa entre estradiol e pulsatilidade de LH;

2. Niveis elevados de $b$-endorfina os quais seriam inibidores do fator hipotalâmico de liberação de gonadotrofinas;

3. Diminuição geral da função hipofisária no obeso acompanhando o conhecido decréscimo de GH e ACTH nestes pacientes; $\mathrm{c}$

4. A observação de que a amplitude do pulso de LH juntamente com a resposta normal das células de Leydig ao estímulo com gonadotrofina coriônica, já mencionados, sugerem que o decréscimo dos níveis séricos de testosterona livre em homens obesos seriam uma conseqüência do hipogonadotrofismo (central).

Sendo assim, decidimos verificar qual a repercussão que a perda de peso traria nos níveis de insulina, testosterona total (TT), testosterona livre (TL) e LH em pacientes com obesidade moderada e severa.

\section{PACIENTES E MÉTODOS}

\section{Pacientes}

Um grupo de homens obesos, idade entre 20-68 anos, foi selecionado para este estudo. Foram divididos em dois grupos: Grupo $1(\mathrm{n}=14)$ com IMC (Índice de Massa Corporal, $\mathrm{kg} / \mathrm{m}^{2}$ ) $\leq 35 \mathrm{~kg} / \mathrm{m}^{2}$; Grupo $2(\mathrm{n}=23) \mathrm{com} I M C \geq 35,1 \mathrm{~kg} / \mathrm{m}^{2}$. Ambos os grupos, em avaliação clínica e laboratorial, mostraram função tireóidea, hepática e renal normais, bem como níveis de glicemia dentro da normalidade. Dois pacientes apresentaram hipertensão moderada e estavam sob uso de diuréticos. Em todos os pacientes obesos avaliaram-se níveis séricos de insulina, testos- terona total, testosterona livre e LH. Os pacientes foram instruídos a seguir dieta de $1200 \mathrm{Kcal} / \mathrm{dia}$, exercícios diários dentro dos limites de suas capacidades e consulta clínica a cada dois meses. Todos os pacientes receberam prescrição de dexfenfluramina ( 1 comprimido de $15 \mathrm{mg} 2$ vezes ao dia). Não houve queixa de marcantes efeitos colaterais com a medicação indicada, exceto sonolência (12\%) e boca seca $(7 \%)$. Após seis meses repetiram-se os testes laboratoriais como descritos acima e os dados clínicos (perda de peso, IMC e pressão sangüínea) foram comparados aos níveis basais.

\section{Controles}

Vinte homens normais, idade entre $24-46$ anos, com IMC $<25 \mathrm{~kg} / \mathrm{m}^{2}$, sem sinais ou sintomas de distúrbio endócrino (mais especificamente a função gonadal), foram selecionados como controles. Testes de rotina bioquímica, testes de função tireóidea, e níveis de gonadotrofina $(\mathrm{LH})$, insulina sérica e testosterona total e livre apresentavam-se normais.

\section{Métodos}

A testosterona total (TT) e a testosterona livre (TL) foram dosadas por RIE (Diagnostic Systems Laboratories, Inc., Webster, TX, USA). A sensibilidade do estojo era de $10,0 \mathrm{ng} / \mathrm{dL}$ e $0,18 \mathrm{pg} / \mathrm{mL}$, respectivamente, $c$ o coeficiente de variação intra $c$ interensaio era de $8,3 \%$ e $7,7 \%$ e $6,2 \%$ e $8,9 \%$, respectivamente. A variação normal de testosterona total é de 280-880ng/dL, a testosterona livre é de 8,7 $48,7 \mathrm{pg} / \mathrm{mL}$.

A insulina foi dosada por radioimunoensaio (Diagnostic Systems Laboratories, Inc., Webster, TX, USA) com sensibilidade de $1,5 \mu \mathrm{U} / \mathrm{mL}$ e coeficiente de variação intra-ensaio de $8,5 \%$ c inter-ensaio $9,9 \%$; valores normais $2,5-30,8 \mu \mathrm{U} / \mathrm{mL}$.

O hormônio luteinizante foi obtido por FIA (Auto DELFIA $n L H$, Wallac OY, Turku, Finland) com limite de sensibilidade de $0,15 \mathrm{mlU} / \mathrm{mL}$ e coeficientes de variação intra e inter-ensaio de $6,6 \% \mathrm{e}$ $7,3 \%$ respectivamente; valores do normal 1,0 $8,4 \mathrm{mlU} / \mathrm{mL}$.

\section{RESULTADOS}

Os resultados são apresentados nas Figuras 1, 2 e 3 e Tabelas 1 e 2 . No Grupo 1 , a média \pm DP do peso que inicialmente era de $96,0 \pm 10,0 \mathrm{~kg}$, declinou para 80,5 $\pm 11,0 \mathrm{~kg}$ ao final de 6 meses de dieta e medicação (variação de perda de peso: $3,2 \mathrm{~kg}$ até $15,0 \mathrm{~kg}$ ). Conse- 


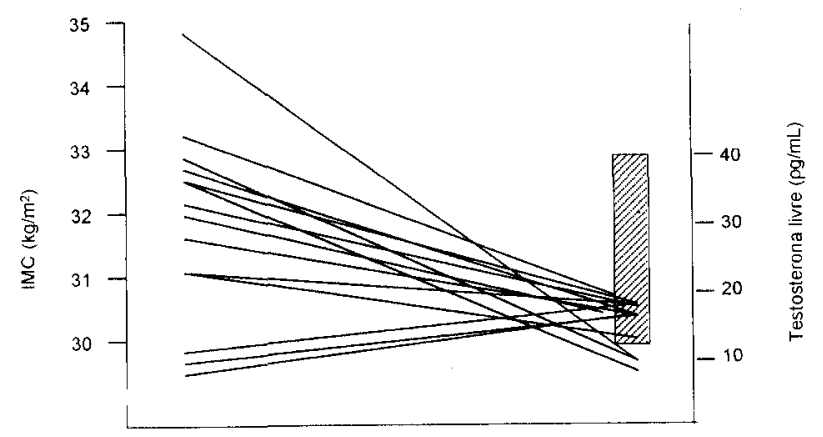

Figura 1. Homens obesos com $I M C \leq 35,0 \mathrm{~kg} / \mathrm{m}^{2}$. A figura mostra o IMC em relação aos niveis séricos de testosterona livre (TL). A barra representa a variação normal da TL

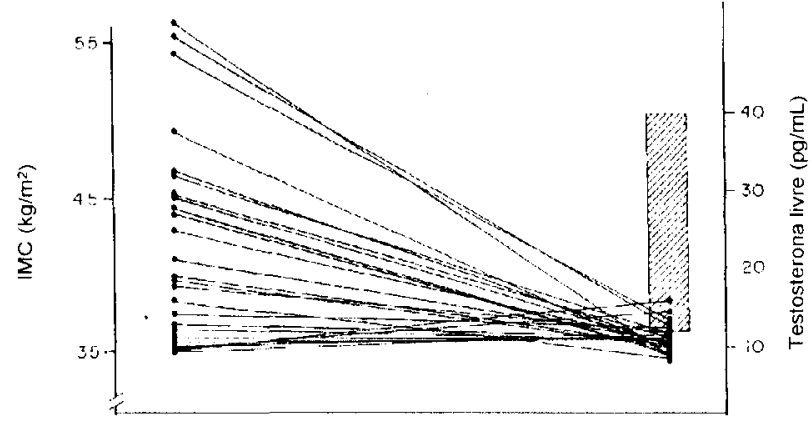

Figura 2. Homens obesos com $I M C \geq 35,1 \mathrm{~kg} / \mathrm{m}^{2}$. Nota-se que, aproximadamente dois terços dos pacientes apresentam baixa concentração sérica de testosterona livre. A barra representa a variação do normal de TL quentemente, o IMC decresceu de $32,3 \pm 1,9$ para $29,4 \pm 2,2 \mathrm{~kg} / \mathrm{m}^{2}$.

Observou-se expressiva diminuição nos níveis do colesterol total, LDL colesterol e triglicérides (resultados não mostrados). A insulina sérica sofreu um decréscimo significativo $(\mathrm{p}<0,05)$ de $46,3 \pm 30,1$ para $19,6 \pm 9,9 \mu \mathrm{U} / \mathrm{mL}$. O hor mônio luteinizante $(\mathrm{LH})$ exibiu um incremento sérico significativo $(\mathrm{p}<0,05)$ em relação aos níveis basais, $4,5 \pm 2,9 \mathrm{mLU} / \mathrm{mL}$ para $6,1 \pm 1,9 \mathrm{mLU} / \mathrm{mL}$ Os níveis séricos de testosterona total e testosterona livre mostraram um aumento significativo $(\mathrm{p}<0,01)$ (Tabela 1$)$.

Os homens obesos que constituíam o Grupo 2 apresentavam peso médio $\pm \mathrm{DP}$ de $131 \pm 25 \mathrm{~kg}$ e, após 6 meses de dieta e medicação, exibiram peso médio \pm DP de $120 \pm 21 \mathrm{~kg}$ (variação de $4,4 \mathrm{~kg}$ até $28,0 \mathrm{~kg}$ ). Como se pode observar na Tabela 2 , verificou-se uma queda significativa dos níveis séricos de insulina $(\mathrm{p}<0,05)$ de $58,1 \pm 23,0$ para $25,4 \pm 7,3 \mathrm{U} / \mathrm{mL}$. O LH sérico aumentou significativamente $(\mathrm{p}<0,05)$ de $3,1 \pm 13 \mathrm{~mL} / \mathrm{mL}$ para $5,0 \pm 2,2 \mathrm{~mL} / \mathrm{mL}$. Como no Grupo 1 , tanto os níveis séricos de testosterona total como a testosterona livre aumentaram significativamente $(\mathrm{p}<0,01)$ após a perda de peso (Tabela 2 , Figura 3). É importante mencionar que os pacientes do Grupo 2 que apresentaram os maiores valores do
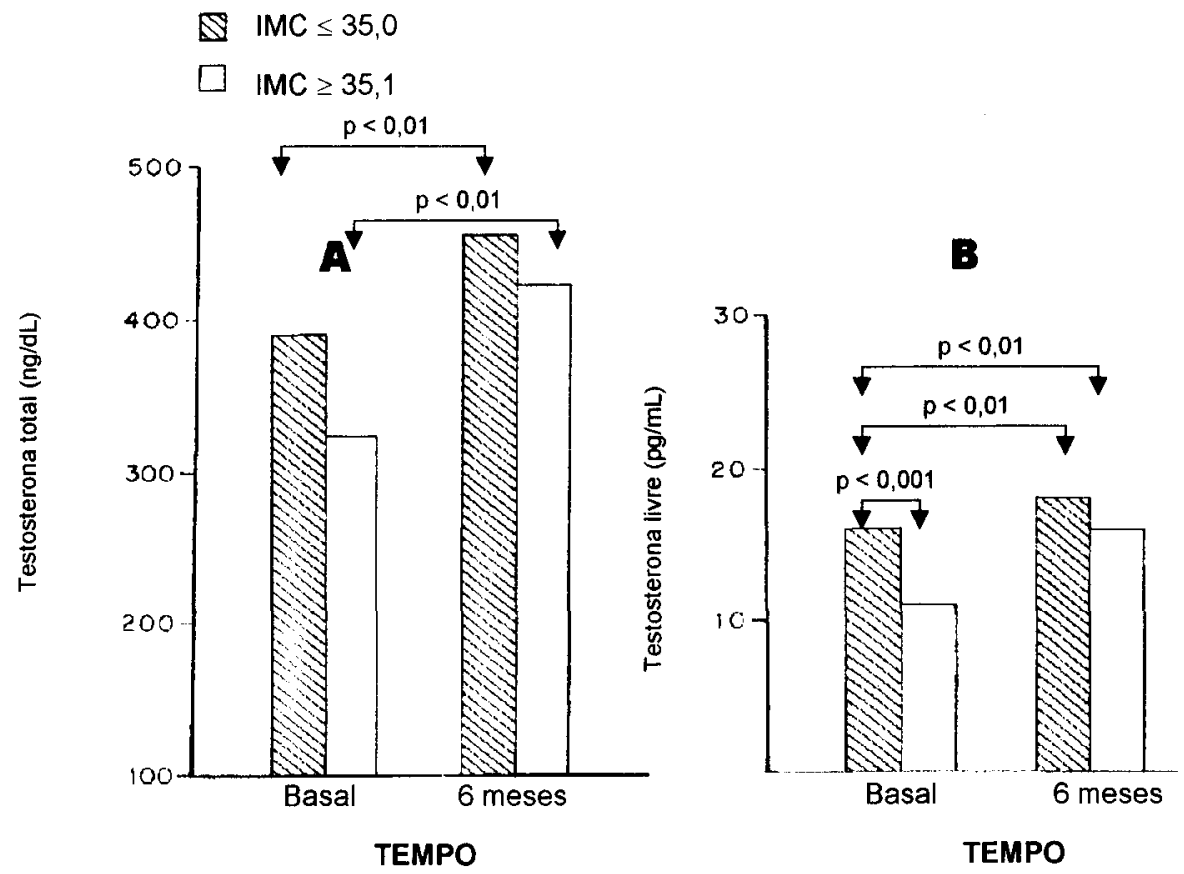

Figura 3. Testosterona sérica total (painel A) e niveis de testosterona livre (painel B) em homens obesos com IMC $\leq 35,0$ $\mathrm{kg} / \mathrm{m}^{2}(\square)$ e $I M C \geq 35,1(\square)$ antes e 6 meses após dieta de baixa caloria e perda de peso 
TABELA 1 -Níveis de andrógenos séricos, LH e insulina (Média \pm DP) em homens obesos (IMC $\leq 35,0 \mathrm{~kg} / \mathrm{m}^{2}$ ) antes e após perda de peso

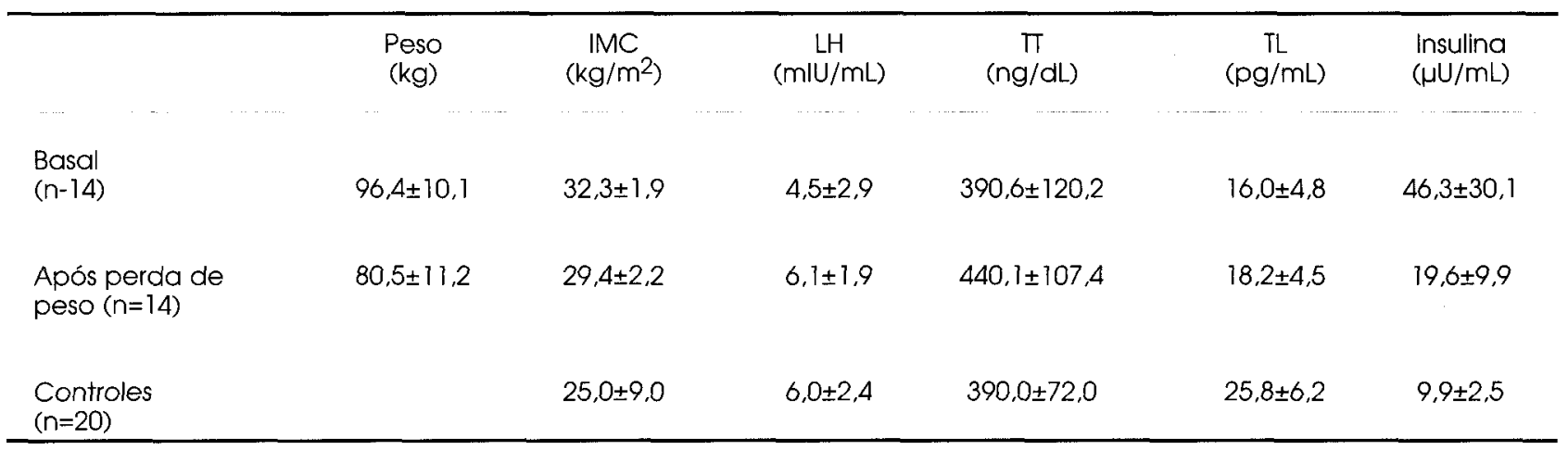

TABELA 2 - Níveis de andrógenos séricos, LH e insulina (Meedia \pm DP) em homens obesos (IMC $\geq 35,1 \mathrm{~kg} / \mathrm{m}^{2}$ ) antes e após perda de peso

\begin{tabular}{|c|c|c|c|c|c|c|}
\hline & $\begin{array}{l}\text { Peso } \\
(\mathrm{kg})\end{array}$ & $\underset{\left(\mathrm{kg} / \mathrm{m}^{2}\right)}{\mathbb{I M C}}$ & $\begin{array}{c}\mathrm{LH} \\
(\mathrm{mlU} / \mathrm{mL})\end{array}$ & $\prod_{(\mathrm{ng} / \mathrm{dL})}$ & $\begin{array}{c}\mathrm{TL} \\
(\mathrm{pg} / \mathrm{mL})\end{array}$ & $\begin{array}{l}\text { Insulina } \\
(\mu U / m L)\end{array}$ \\
\hline & & & & & & \\
\hline $\begin{array}{l}\text { Basal } \\
(n-24)\end{array}$ & $131 \pm 25,6$ & $43,0 \pm 6,7$ & $3,1 \pm 1,3$ & $320 \pm 110$ & $11,0 \pm 2,1$ & $58,1 \pm 23,0$ \\
\hline $\begin{array}{l}\text { Após perda de } \\
\text { peso }(n=24)\end{array}$ & $120,0 \pm 21,0$ & $38,0 \pm 5,5$ & $5,0 \pm 2,2$ & $420 \pm 56$ & $16,0 \pm 4,4$ & $25,4 \pm 7,3$ \\
\hline $\begin{array}{l}\text { Controles } \\
(n=20)\end{array}$ & & $25,0 \pm 9,0$ & $6,0 \pm 2,4$ & $390 \pm 72$ & $25,8 \pm 6,2$ & $9,9 \pm 2,5$ \\
\hline
\end{tabular}

Índice de Massa Corporal tinham os menores valores de testosterona total na situação basal, bem como após 6 meses de tratamento.

$\mathrm{Na}$ Figura 3 (painel B), pode-se verificar em destaque, a variação na concentração da testosterona livre. Podemos verificar que os pacientes com os maiores valores de IMC $\left(\geq 35,1 \mathrm{~kg} / \mathrm{m}^{2}\right)$ apresentam significativamente $(\mathrm{p}<0,01)$ os menores níveis médios séricos de testosterona livre quando comparados aos do Grupo 1 na situação basal.

Após perda de peso (em 6 meses), ambos os grupos mostraram um aumento significativo $(\mathrm{p}<$ $0,01)$ nos níveis de testosterona total. Notar que o Grupo 2 mostrou menor incremento do nível médio de testosterona livre quando comparado ao Grupo 1 (Figura 3).

\section{DISCUSSĀO}

O presente estudo confirmou que, em homens obesos, os níveis séricos de testosterona são significativamente menores quando comparados a homens normais da mesma idade. Verificamos na literatura (27) que a concentração de SHBG apresenta correlação linear negativa com o IMC e correlação linear positiva entre insulina e IMC. Estudo experimental já havia demonstrado o efeito inibitório da insulina na síntese de SHBG (17). Consequentemente, a redução dos níveis de testosterona total em homens é ligada ao decréscimo dos níveis de SHBG, os quais são condicionados aos altos níveis de insulina circulante. Verificamos, igualmente, que os níveis médios (média $\pm \mathrm{DP}$ ) de testosterona total que se mostravam diminuídos nos homens obesos não mostram diferença significativa entre o grupo com obesidade moderada (IMC 32,3 \pm $1,9 \mathrm{~kg} / \mathrm{m}^{2}$ ) quando comparados ao grupo com obesidade severa (IMC $43,0 \pm 6,7 \mathrm{~kg} / \mathrm{m}^{2}$ ). A mesma constatação estatística foi verificada quando analisamos a concentração dos níveis séricos médios $( \pm \mathrm{DP})$ de insulina (respectivamente $46,3 \pm 30,1 \mu \mathrm{U} / \mathrm{mL}$ e 58,1 $\pm 23,0 \mu \mathrm{U} / \mathrm{mL})$ nos dois grupos de pacientes. Entretanto, homens com obesidade moderada apresentavam um aumento sérico médio $( \pm \mathrm{DP})$ significativo $(\mathrm{p}<$ $0,01)$ de testosterona livre $(16,0 \pm 4,8 \mathrm{pg} / \mathrm{mL})$ quan- 
do comparado com homens de obesidade de grande porte $(11,0 \pm 2,1 \mathrm{pg} / \mathrm{mL})$. Isto pode ser devido ao nível sérico baixo de $\mathrm{LH}$, bem como a menor amplitude de pulso de LH como já foi descrito em homens com obesidade severa, implicando uma possível alteração da regulação gonadostática (31). Após 6 meses de perda de peso, observou-se um significativo aumento nos níveis séricos de TT, TL e LH que se correlacionaram com a diminuição da concentração de insulina plasmática. Observou-se, em estudos pregressos, que a perda de peso por si só, pode elevar os níveis de SHBG. Strain et al. (32) mostraram, recentemente, que durante a perda de peso, os níveis séricos de SHBG aumentaram em média $0,43 \mathrm{nmol} / \mathrm{L}$ por cada unidade de diminuição do IMC. Sendo assim, após a perda de peso, o aumento da concentração sérica de testosterona total poderia ocorrer por duas diferentes vertentes: (1) por aumento da capacidade de ligação do SHBG e (2) por aumento da amplitude dos pulsos espontâneos de LH. O decréscimo dos níveis de TL, somente encontrado em homens com severa obesidade, sugère menor estimulação das células de Leydig devido a alteração funcional do gonadostato. Adicionalmente, conforme resultados publicados muito recentemente (33), pode-se admitir que os níveis elevados de leptina podem ser um fator importante na redução de andrógenos circulantes no homem obeso. Este hipoandrogenismo resultante é bem documentado laboratorialmente mas surpreendentemente apresenta pequena repercussão clínica, seja na libido como na atividade sexual.

Na nossa casuística, somente dois pacientes se queixaram de diminuição de libido e menor atividade sexual. Todos os demais pacientes tinham comportamento sexual normal e não notaram diferença antes e após a perda de peso. É admissível que a diminuição dos níveis séricos de TL em alguns pacientes bem abaixo da variação do normal esteja ainda dentro dos níveis fisiológicos sem nenhum reflexo na sexualidade do paciente.

Em conclusão, demonstramos que homens com obesidade de grande porte, IMC acima de $35,1 \mathrm{~kg} / \mathrm{m}^{2}$ têm níveis séricos de testosterona livre reduzidos que melhoram após a perda de peso. Além disso, a diminuição da concentração de insulina plasmática a níveis quase normais levam à normalização dos níveis de SHBG e TT após a perda de peso.

\section{REFERÊNCIAS}

1. Glass AR, Swerdioff RS, Bray GA, Dahms W, Atkinson RL. Low serum testosterone and sex hormone binding globulin in massively obese men. J Clin Endocrinol Metab 1977:45:1211-9.
2. Stanik S, Dornfeld LP, Maxwell MH, Viosca SP, Korenman SG. The effect of weight loss on reproductive hormones. $\mathrm{J}$ Clin Endocrinol Metab 1981:53:828-31.

3. Glass AR, Burman KD, Dahms WT, Boehm TM. Endocrine function in obesity. Metabolism 1981:30:89-104.

4. Vermeulen A, Kaufman JM, Deslijpere JP, Thomas $G$. Attenuated luteinizing hormone (LH) pulse amplitude but normal LH pulse frequency, and its relation to plasma androgens in hypogonadism of obese men. J Clin Endocrinol Metab 1993;76:1 140-6

5. Amatruda JM, Harman SM, Pourmotabbed G, Lockwood DH. Depressed plasma testosterone and fractional binding of testosterone in obese males. J Clin Endocrinol Metab 1978:47:268-71.

6. Strain $G$, Zumoff B, Miller IK, Rosenfeld RS. The influence of massive weight loss in the hypogonadotropic hypogonadism of obese men Abstract Int J Obes II (Supp| 2) 1987:54.

7. Kley HK, Kruskemper HL. Gesamt und freles testosteron beim mann in Abhangigkeit vom Korpergewicht. Deutsch Med Wochenschr 1979:42:1478-82.

8. Zumoff B, Strain GW. Miller LK, Rosner W, Senie R, Seres DS et al. Plasma free and non sex hormone binding globulin bound testosterone are decreased in obese men in proportion to their degree of obesity. J Clin Endocrinol Metab 1990;71:929-31.

9. Pasquali R, Macor C, Viconnati V. Novo F. De lasio R, Mesini $P$ et al. Effects of acute hyperinsulinemia on testosterone serum concentrations in adult obese and normal-weight men. Metabolism 1997:46(5): 526-9.

10. Sydell JC, Björtor P, Sjoström L, Sammerstedt T. Visceral fat accumulation in men is positively associated with insulin, glucose and c-peptide levels, but negatively with testosterone levels. Metabolism 1990; 33(9):897-901.

11. De Moor $P$, Joosens $G V$. An inverse relationship between body weight and the activity of the steroid binding b-globulin in human plasma. Steroidologia 1970:2:129-36.

12. Vermeulen A. Testosterone in plasma. In: Advances in Biosciences $2^{\text {nd }}$ ed. Berlin, New York: W De Gruyter Raspe. 1968:105-22.

13. Vermeulen A, Verdonck L, Van der Straeten M, Orie N. Capacity of the testosterone binding globulin in human plasma and influence of specific binding of testosterone on its metabolic clearance rate. J Clin Endocrinol Metab $1969 ; 29: 1470-80$.

14. Kahn MS, Ewen E, Rosner W. Radioimmunoassay for human testosterone-estradiol-binding globulin. J Clin Endocrinol Metab 1992;54:705-9.

15. Hryb DJ, Khan MS, Rosner W. Testosterone-estradiolbinding globulin binds to human prostatic cell membranes. Biochem Blophys Res Commun 1985:128: $432-40$.

16. Rosner W, Hryb DJ, Khan MS, Nakla AM, Romas NA. Sex hormone-binding globulin. Binding to cell membranes and generation of a second messenger. J Androl 1992; 13:101-6. 
17. Plymate SR, Coop SM, Hoop RC et al. Effects of sex hormone binding globulin (SHBG) on human prostatic carcinoma. J Steroid Biochem Mol Biol $1991: 40: 833-9$

18. Schneider $G$, Kirschner MA, Berkowitz R et al. Increased estrogen production in obese men. J Clin Endocrinol Metab 1979:48:633-8.

19. Marin $P$, Holmang $S$, Jonssen $L$ et al. The effects of testosterone treatment in body competition and metabolism in middle-aged obese men. Int J Obesity $1992 ; 16: 991-7$.

20. Barrett-Connor $E$, Laaksen $M$. Ischemic heart disease risk in postmenopausal women. Effects of estrogen use on glucose and insulin levels. Arteriosclerosis 1990:10:531-4.

21. Plymate SR, Matij LA, Jones RE, Fried/ KE. Inhibition of sexhormone-binding globulin production in the human hepatoma (Heo 72) cell line by insulin and prolactin. $J$ Clin Endocrinol Metab 1988;67:460-4.

22. Haffner SM, Katz MS, Stern MP, Dunn JF. The relationship of sex hormones to hyperinsulinemia and hyperglycemia. Metabolism 1988;37:683-8.

23. Zumoff B. Hormonal abnormalities in obesity. Acta Med Scand 1988:723 (Suppl): 153-60.

24. Pasquali $R$, Casimirri $F$, Cantobelli $S$, Melchionda $N$ Labate AMM, Fabbri $R$ ef al. Effect of obesity and body fat distribution on sex hormones and insulin in men. Metabolism 1991:40:101-4

25. Birkeland KI, Hanssen KF, Torjesen PA et al. Level of sexhormone-binding globulin is positively correlated with insulin sensitivity in men with type 2 diabetes. J Clin Endocrinol Metab 1993:76:275-8.

26. Bagdade JD, Porte Jr D, Brunzell JD et al. Basal and stimulated hyperinsulinism: reversible metabolic sequel of obesity. J Lab Clin Med 1973;83:563-9.
27. Falkner B, Sherif K, Sumner A, Kushner H. Hyperinsulinism and sex hormones in young adult African Americans. Metabolism 1999:48:107-12.

28. Amatruda JM, Harman SM, Pourmotabbed G. Hypothalamic and pituitary dysfunction in obese males. Int $J$ Obes 1982;6: 183-9.

29. Amatruda JM, Hochstein M, Hsu TH et al. Testosterone deficiency of morbid obesity. Possible role of the hypothalamus. Clin Res 1975;23:571A.

30. Surynarayana BV, Kent JR, Meister L et al. Pituitarygonadal axis during prolonged total starvation in obese men. Am J Clin Nutr 1969:22:767-70.

31. Giagulli VA, Kaufman JM, Vermeulen A. Pathogenesis of the decreased androgen levels in obese men. J Clin Endocrinol Metab 1994;79:997-1000.

32. Strain G, Zumoff B, Rosner W, Pi-Sunyer X. The relationship between serum levels of insulin and sex hormone-binding globulin in men: the effect of weight-loss. J Clin Endocrinol Metab 1994;70:1173-6.

33. Isidori AM, Caprio M, Strollo F, Moretti C, Frajese G, Isidori $A$, et al. Leptin and androgens in male obesity: evidence for leptin contribution to reduced androgen levels. J Clin Endocrinol Metab 1999:84:3673-80.

\section{Endereço para correspondência:}

Geraldo Medeiros-Neto

Hospital das Clinicas

Av. Dr. Enéas C. Aguiar 155, 8A, bl. 3

05403-900 São Paulo, SP

Fax: (55 11) 211-5194

Fone Fax: $(5511) 3064-6835$

e-mail: medneto@uol.com.br 